\section{Atypical femur fractures asso- ciated with bisphosphonates: from prodrome to resolution}

\author{
Rafael Sanjuan-Cervero, ${ }^{1}$ \\ Braulio Sastre-Jala, ${ }^{2}$ \\ Elaine Heredia-Heredia, ${ }^{3}$ \\ Nuria Franco-Ferrando, ${ }^{1}$ \\ Jaime Poquet-Jornet ${ }^{4}$ \\ 'Orthopedic Surgery and Traumatology \\ Service, ${ }^{2}$ Internal Medicine Service, \\ ${ }^{3}$ Family and Community Medicine, \\ ${ }^{4}$ Clinical Department of Pharmacy, \\ Hospital of Dénia, Alicante, Spain
}

\begin{abstract}
Atypical fractures related to the prolonged use of bisphosphonates are caused by low energy mechanisms and are characterized by oblique and transverse lines and frequent bilateralism. We present a clinical case of a patient who we believe illustrates, both in clinical and radiological aspects, the new definition of atypical femur fracture related to treatment using bisphosphonates treated conservatively and successfully with discharge and teriparatide $20 \mathrm{mcg} / 80 \mathrm{mcl} \mathrm{s.c} / 24 \mathrm{~h}$. The appearance of painful symptoms in the upper thigh, especially if bilateral, in patients treated with bisphosphonates for long periods of time, makes it necessary to dismiss bone lesions that might otherwise suggest atypical fracture. In those cases where the fracture is incomplete, restoring bone metabolism through the administration of teriparatide $20 \mathrm{mcg} / 80 \mathrm{mcl}$ s.c./24h could prevent displaced fractures.
\end{abstract}

\section{Introduction}

Bisphosphonates have been widely used in the treatment of osteoporosis for years, to prevent the clinical outcomes of the condition, fragility fractures, as they have been shown to be highly effective and to have an excellent safety profile. However, as with any other drug, they are not devoid of side effects, and they have been associated with cardiovascular events, such as atrial fibrillation, and maxillary osteonecrosis. ${ }^{1}$

Since 2005, a possible association between bisphosphonate use and the appearance of atypical stress fractures, ${ }^{2}$ both in clinical presentation (rare, caused by a low impact traumatism or even with no traumatism, painful prodromes preceding the fracture by weeks or months, and frequent bilateralism), and radiological presentation (transverse trace or with minimal obliquity), has been noted. Since that time, several studies, have presented a series of conclusions, some of them contradictory, regarding this possible relationship..$^{3-5}$

There have been two main problems facing investigators: first, the lack of any clear definition of what an atypical fracture is, and, second, that for many of the assessments performed, the selection of patients was based on admission codes, without taking radiological data into account. Thus, the proportion of patients with diaphyseal or subtrochanteric fractures that could actually be considered atypical was not known. ${ }^{6}$

In 2010, the American Society for Bone and Mineral Research (ASBMR) task force published a provisional recommendation for defining a typical case based on a series of major and minor criteria, which included various radiological characteristics. ${ }^{6}$ Such guidelines have allowed for the publication of meaningful studies, ${ }^{7}$ following the recommended definition, that have helped to clarify the picture, and to strengthen acceptance of the aforementioned association between the extended use of bisphosphonates and the appearance of atypical femur fractures. Furthermore, it has been shown that the incidence of atypical fractures related to treatment with bisphosphonates is rather low, and that the risk-benefits ratio for bisphosphonate use is quite favorable. Publication of these studies has led investigators to reconsider the roles that other processes, or treatments, could have in the appearance of atypical fractures, which has recently led the ASBMR task force to take a fresh look at its provisional recommendation for defining a typical case.

According to the revised case definition, the fracture must be located along the femoral diaphysis with requirement of at least four of the following 5 major features: i) association with minimal or no trauma, ii) fracture line originating at the lateral cortex with substantial transverse or oblique orientation, iii) extension of complete fractures through both cortices with or without a medial spike or involvement only of the lateral cortex for incomplete fractures, iv) non-comminution or minimal comminution, v) presence of localized periosteal or endosteal thickening of the lateral cortex at the fracture site. ${ }^{8}$

\section{Case Report}

A 71-year-woman was admitted to our hospital for a diaphyseal fracture of the right femur with short oblique trace and no comminution. The fracture occurred while she was in a standing position after she turned her leg slightly with her foot in a fixed position on the floor. She had not experienced trauma or a pre-
Correspondence: Rafael Sanjuan Cerveró, Calle del Vall 42, 1 Cullera 46400, Valencia, Spain. Tel.:+34.6555.23150.

E-mail: sanjuan.rafcer@gmail.com

Key words: Bisphosphonates; atypical fracture; stress fracture.

Contributions: RSC and BSJ created the conception and design of the article; EHH reviewed the literature and started the draft of the project; NFF and RSC made the follow up of the report and started with the original manuscript; JPJ reviewed the article and suggested modifications and a new point of view in the treatment of this case.

Conflict of interest: the authors declare no potential conflict of interest.

Received for publication: 8 June 2015 .

Revision received: 22 July 2015 .

Accepted for publication: 22 July 2015.

This work is licensed under a Creative Commons Attribution NonCommercial 3.0 License (CC BYNC 3.0).

CCopyright R. Sanjuan-Cervero et al., 2015

Licensee PAGEPress, Italy

Rheumatology Reports 2015; 7:6068

doi:10.4081/rr.2015.6068

vious fall (Figure 1A). The patient reports pain in both inguinal regions with irradiation from the internal side of the thighs during the last 5 months. Her condition was considered secondary to bilateral coxarthrosis and treated with non-steroidal anti-inflammatory drugs. The pathological background of interest that she mentions is arterial hypertension, hypercholesterolemia and osteoporosis. A review of her pharmacotherapeutic history documents treatment with alendronate $70 \mathrm{mg} / \mathrm{calciferol} 5600 \mathrm{U}$ weekly during the last 5 years.

Based on her clinical background, a diagnosis of suspected atypical femoral fracture associated with bisphosphonate use (alendronate) was established. Therefore, radiological exploration of the contralateral femur was performed, which showed an incomplete linear fracture of the femoral cortex at the middle third of the external femoral diaphysis (Figure 2). A determination of hematometric and biochemical parameters established the levels of calcidiol $(22.6 \mathrm{ng} / \mathrm{mL})$, alkaline phosphatase (129 U/L), calcium $(8 \mathrm{mg} / \mathrm{dL})$, phosphorous $(3.8 \mathrm{mg} / \mathrm{dL})$, and parathyroid hormone $(70.4$ $\mathrm{pg} / \mathrm{mL}$ ). No significant alterations were observed, except for slightly low levels of calcidiol and a very discrete elevation of intact PTH levels. Three months before suffering this fracture, the patient had undergone a densito- 
metric study with a -2.3 T-Score and $-0.8 \mathrm{Z}$ Score for the right femoral neck and a $-3.9 \mathrm{~T}$ Score and $-1.7 \mathrm{Z}$-Score for the lumbar spine. The secondary effects of alendronate were determined through a modified Karch-Lasagna algorithm, considering the secondary effect as probable. ${ }^{9}$

The patient was treated for the displaced right femoral fracture using intramedullary nailing with Gamma3 ${ }^{\circledR}$ (Stryker Inc., Kalamazoo, MI, USA) nails with double distal locking and not bearing weight (Figure 1B). After the two options of a conservative approach or surgical treatment were discussed with the patient, and the risks and benefits of each option were explained, conservative treatment with not bearing weight and teriparatide $20 \mathrm{mcg} / 80 \mathrm{mcl}$ s.c. $/ 24 \mathrm{~h}$ was selected.

The patient remained in the non-weight bearing stage for six weeks after which progressive weight bearing was authorized for both limbs. After six months, the patient was asymptomatic at the fracture regions, could walk without help, and reported only occasional discomfort after prolonged walking in the right greater trochanter region due to placement of the intramedullary nail, and with radiological resolution of both fractures (Figure 3 ). Treatment with teriparatide $20 \mathrm{mcg} / 80 \mathrm{mcl}$ s.c./24h was maintained for a total of 24 months in order to restore her bone mineral metabolism and improve bone quality.

\section{Discussion}

The use of bisphosphonates in the treatment of osteoporosis for preventing fragility fractures is well-established and universally accepted in all clinical guides. Bisphosphonates are currently the first-line treatment for post-menopausal osteoporosis. ${ }^{8}$ However, experience after all these years using these drugs, has shown that undesirable renal, cardiovascular, digestive and bonemetabolic side effects are associated with their use. ${ }^{1}$ Adverse events on bone derive from the drugs' mechanism of action. Nitrogenous bisphosphonates like alendronate bind to and inhibit the activity of farnesyl pyrophosphate synthase, leading to osteoclast apoptosis and suppression of bone remodeling which, in the long-term, favors stress fractures. ${ }^{10}$ On the other hand, their affinity for bone tissue allows them to stay in the bone, once they have been fixed, until their elimination with a half-life of 10.9 years. ${ }^{11}$

The suspicion of association between the use of bisphosphonates and the appearance of atypical femur fractures was already noted in 2005 by Odvina. $^{2}$ Since then, several studies have confirmed the existence of this association, ${ }^{3-5}$ although it is still considered a very

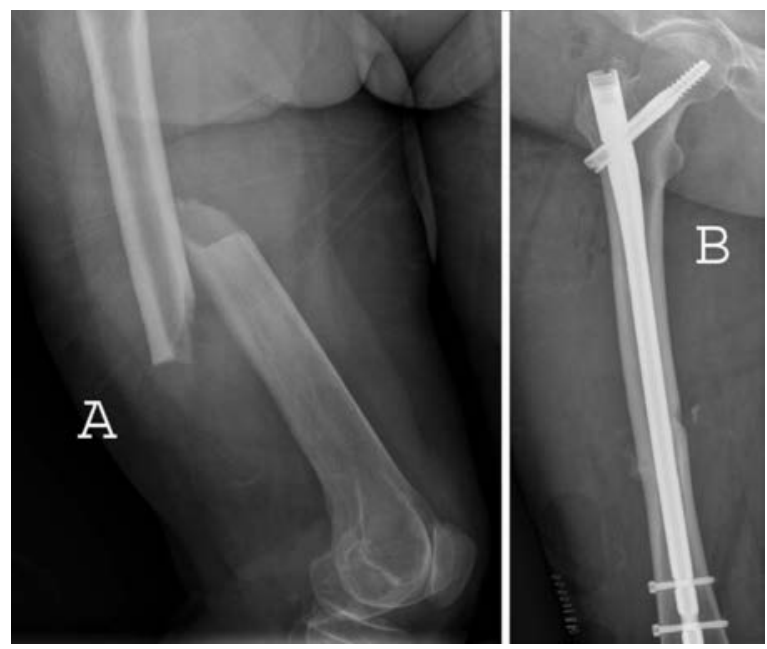

Figure 1. Right femur: A) detail of the fracture at admittance; B) treatment with intramedullary nail.

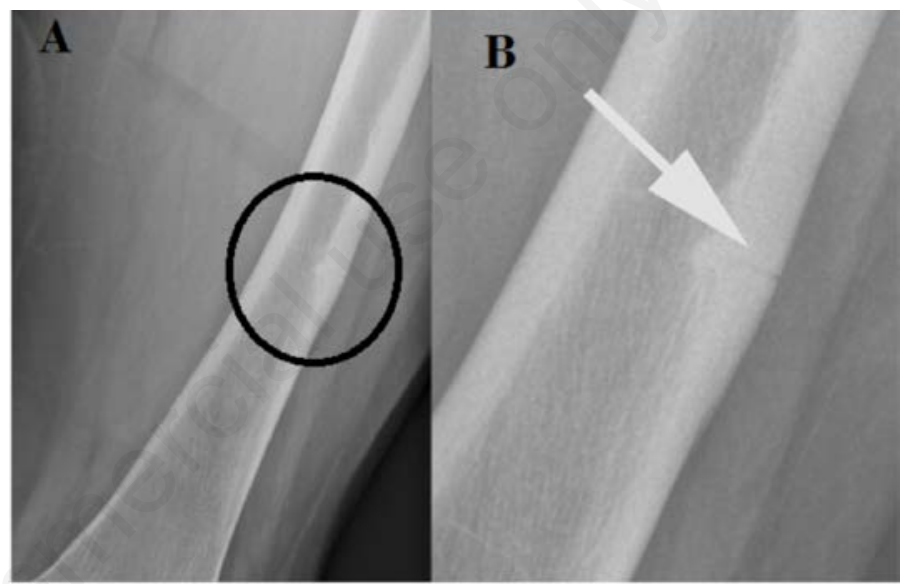

Figure 2. Left femur: A) detail of atypical fracture; B) zoom in to the affected area, where the linear trace caused by the stress fracture (arrow head) and the widening of the outer cortex with respect to the inner cortex, can be observed.

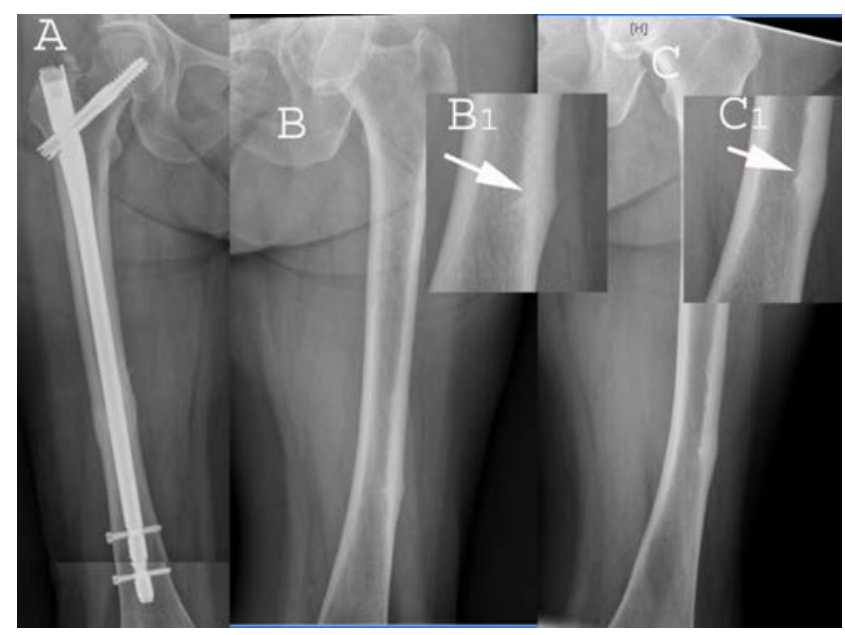

Figure 3. A) Evolution of right femur at 6 months with complete resolution of the fracture. B) Six months evolution of the left femur with progression of the remodelation of the lateral cortex. B1) Zoom in to the affected area where the line of fracture has disappeared, but maintaining cortex irregularity. C) One year evolution of the left femur. C1) Zoom in to the affected area where it can see progressive normalization of the cortex. 
rare occurrence, and so the risk-benefits ratio for these drugs is still considered favorable.

However, since the appearance of atypical fractures is associated with extended treatment with bisphosphonates, consensus of opinion is leaning more and more towards limiting the duration of treatment, although it has not yet been established how long treatment with these drugs may be safely used, and a time out period called therapeutic holiday after 5 years of continuous administration is recommended if there are no clear risk factors for the appearance of osteoporotic fractures. ${ }^{12,13}$

In the case of our patient, the clinical fracture took place just after finishing five years of treatment and, if the bilateral inguinal pain is assumed to be prodromal, the period of treatment should probably have been cut after 3-4 years. The difference between our clinical case and other previously published cases is the clear and precise definition of the clinical characteristics preceding the detection of the atypical fracture. In the majority of the cases symptoms last for months and may lead to a wrong diagnosis of degenerative processes. ${ }^{14}$ Once the fracture is established, treatment is diaphyseal intramedullary nailing. Regarding the need for prophylactic treatment for atypical fractures, the criteria are not well-established. Lee recommends, ${ }^{15}$ in his study of some 65 fractures of this type, performing prophylactic nailing when there is subtrochanteric localization, as do other authors. ${ }^{16}$ On the other side, there is also evidence supporting a conservative treatment option, ${ }^{17-19}$ such as pharmacological therapy with teriparatide $20 \mathrm{mcg} / 80 \mathrm{mcl}$ s.c./24h. This approach may contribute to increase bone turnover, restoring bone metabolism and preventing delays in the consolidation of this type of fracture. ${ }^{17}$ Nevertheless, the ASBMR finds inconsistent scientific evidence for healing time acceleration for these fractures because of the administration of teriparatide. ${ }^{8}$

\section{Conclusions}

At all events, the appearance of painful symptoms in the upper part of the thigh, especially when bilateral, in patients treated with bisphosphonates over a long period of time, makes a detailed radiological study, including both complete femur bones, in order to rule out bone lesions suggesting atypical fracture, a must. Once the fracture is established, treatment is mainly surgical, with the objective of reducing and stabilizing the fracture. But in those cases where the fracture is incomplete, restoring bone metabolism using teriparatide may prevent the patient from suffering complete fracture. Finally, the so-called therapeutic holiday, interrupting the administration of bisphosphonates in those cases where no contraindications exist, or where the effect of the withdrawal doesn't involve an increase in the risk of fracture, also plays a fundamental role in preventing such complications.

\section{References}

1. Arboleya L, Alperi M, Alonso S. Efectos adversos de los bisfosfonatos. Reumatol Clin 2011;7:189-97.

2. Odvina CV, Zerwekh JE, Rao DS, et al. Severely suppressed bone turnover: a potential complication of alendronate therapy. J Clin Endocrinol Metab 2005;90:1294-301

3. Abrahamsen B, Eiken P, Eastell R. Subtrochanteric and diaphyseal femur fractures in patients treated with alendronate: a register-based national cohort study. J Bone Miner Res 2009;24:1095-102.

4. Black DM, Kelly MP, Genant HK, et al. Bisphosphonates and fractures of the subtrochanteric or diaphyseal femur. N Engl J Med 2010;362:1761-71.

5. Kim SY, Schneeweiss S, Katz JN, et al. Oral bisphosphonates and risk of subtrochanteric or diaphyseal femur fractures in a population-based cohort. J Bone Miner Res 2011;26:993-1001.

6. Shane E, Burr D, Ebeling PR, et al. Atypical subtrochanteric and diaphyseal femoral fractures: report of a task force of the American Society for Bone and Mineral Research. J Bone Miner Res 2010;25:226794.

7. Schilcher J, Michaëlsson K, Aspenberg P. Bisphosphonates use and atypical fractures of the femoral shaft. N Engl $J$ Med 2011;364:1728-837.
8. Shane E, Burr D, Abrahamsen B, et al. Atypical subtrochanteric and diaphyseal femoral fractures: second report of a task force of the American Society for Bone and Mineral Research. J Bone Miner Res 2014;29:1-23.

9. Karch FE, Lasagna L. Toward the operational identification of adverse drug reactions. Clin Pharmacol Ther 1977;21:24754.

10. Burr DB, Forwood MR, Fyhrie DP, et al. Bone Microdamage and skeletal fragility in osteoporotic and stress fractures. $\mathrm{J}$ Bone Miner Res. 1997;12:6-15.

11. Goddard MS, Reid KR, Johnston JC, et al. Atraumatic bilateral femur fracture in long-term bisphosphonate use. Orthopedics. 2009;32:607-10.

12. Ott SM. Long-term safety of bisphosphonates. J Clin Endocrinol Metab 2005;90:189799.

13. Brown JP, Morin S, Leslie W, et al. Bisphosphonates for treatment of osteoporosis. Can Fam Physician. 2014;60:324-33.

14. Chan SS, Rosenberg ZS, Chan K, et al. Subtrochanteric femoral fractures in patients receiving long-term alendronate therapy: imaging features. Am J Roentgenol 2010;194:1581-6.

15. Lee YK, Ha YC, Kang BJ, et al. Predicting need for fixation of atypical femoral fracture. J Clin Endocrinol Metab. 2013;98:2742-5.

16. Banffy MB, Vrahas MS, Ready JE, et al. Nonoperative versus prophylactic treatment of bisphosphonate-associated femoral stress fractures. Clin Orthop Relat Res 2011;469:2028-34.

17. Carvalho NN, Voss LA, Almeida MO, et al. Atypical femoral fractures during prolonged use of bisphosphonates: short-term responses to strontium ranelate and teriparatide. J Clin Endocrinol Metab 2011;96:2675-80.

18. Saleh A, Hegde VV, Potty AG, et al. Management strategy for symptomatic bisphosphonate-associated incomplete atypical femoral fractures. HSS J 2012;8:103-10.

19. Fukuda F, Kurinomaru N, Hijioka A. Weekly teriparatide for delayed unions of atypical subtrochanteric femur fractures. Biol Ther 2014;4:73-79. 